\title{
Anesthetic Management of a Boy with Congenital Disorder of Glycosylation (CDG) I-X
}

\author{
Amit Lehavi ${ }^{1,3^{*}}$, Hanna Mandel ${ }^{2,3,4}$, Yeshayahu (Shai) Katz ${ }^{1,3,4}$ \\ ${ }^{1}$ Department of Anesthesiology, Rambam Healthcare Campus, Haifa, Israel; ${ }^{2}$ Metabolic Unit, Meyer Children's Hospital, Haifa, \\ Israel; ${ }^{3}$ Rambam Health Care Campus, Haifa, Israel; ${ }^{4}$ Bruce Rappaport Faculty of Medicine, Technion (Israel Institute of Technol- \\ ogy), Haifa, Israel. \\ Email: "amit.lehavi@gmail.com
}

Received April 16 ${ }^{\text {th }}, 2011$; revised April 27 ${ }^{\text {th }}, 2011$; accepted May 27 $7^{\text {th }}, 2011$.

\begin{abstract}
Congenital disorders of glycosylation (CDGs) are group group of genetic defects in the assembly and processing pathway of protein glycosylation, which cause a wide range of multi system dysfunction. This paper describes the anesthetic management of 6 years old boy with CDG type I-x for upper airway surgery. We used a sevoflurane-nitrous oxide-remifentanil regime with no complications and good results. As for now, the literature lacks reports of anesthetic management of children with CDG I-x, and this report may provide clinicians an option for safe anesthetic management.
\end{abstract}

Keywords: Anesthesia, Pediatric, Metabolic Disorders, Congenital Disorders of Glycosylation, CDG

\section{Introduction}

Congenital disorders of glycosylation (CDGs) are group of genetic defects in the assembly and processing pathway of protein glycosylation. This causes a phenotype of which growth, cardiac, neurological, hematological, gastrointestinal, renal, and dermatological and ophthalmological systems are affected in various penetration. The literature concerning information on the anesthetic management of CDG patients is very limited. The purpose of our report is to raise the awareness of anesthesiologists to this group of disorders, with emphasis on the CDG I-x subtype, because specific diagnosis of any entity is important for preventive measures. We describe the anesthetic management of a six years old boy suffering from CDG Type I-x, which was referred for an upper airway surgery under general anesthesia.

\section{Case Presentation}

DS is a 6 years old boy, whose parents are second degree cousins of Druze origin. He was considered as a healthy and normally developing boy until his brother was found to have hypertransaminasemia and dilated cardiomyopathy. These findings led to the screening of all family members including DS who was found to have the same symptoms as his brother including hypertrnasaminasemia and dilated cardiomyopahty at the age of 5 years. The boy, weighing $16.2 \mathrm{~kg}$ (second percentile), had no cognitive impairment, and physical examination did not reveal any abnormal findings. He was put on oral captopril $2.5 \mathrm{mg}$ twice daily. Retrospectively, his medical chart showed a constant finding of hypertransaminasemia since the age of 6 months with alanine aminotransferase, (ALT) up to $387 \mathrm{U} / \mathrm{L}$ (normal values: 5 - $45 \mathrm{U} / \mathrm{L}$ ) and aspartate aminotransferase (AST) rising to $302 \mathrm{U} / \mathrm{L}$ (normal values: 15 - 55 U/L). Serum bili- rubin, alkaline phosphatase and gammaglutamyltrans-peptidase (GGT) were normal, as were total protein, albumin, cholesterol, iron, ferritin, blood count, creatine phosphokinase, electrolytes, urea and creatinine. Further metabolic studies including serum lactate, aminoacids and urinary organic acids were normal. Isoelectric focusing of transferrin was performed because CDGs are considered in the differential diagnosis of hypertransaminasemia and dilated cardiomyopathy. Serum isoelectrofocusing (IEF) of transferrin revealed a type I profile suggesting a glycan assembly defect (CDG-I). However, analysis of the patient's fibroblasts eliminated the possibility of CDG I-a and CDG I-b; lipid-linked oligosaccharide analysis did not show any abnormal accumulation of glycan intermediates. To date, the primary genetic defect of the glycosylation pathway remains unknown, thus we designated the siblings' disorder as CDG I-x. 
The child was scheduled for tonsillectomy and adenoidectomy due to upper airway obstruction. Coagulation studies revealed decreased levels of Protein C, Protein S and Factor XI (26\%, 59\% and 45\% respectively), with normal prothrombin (international normalized ratio 1.26) and partial thromboplastin time $25 \mathrm{sec}$.

\section{Anesthetic Management}

The child was instructed to fast the evening prior to surgery, and did not receive oral premedication. Induction of anesthesia was achieved with a mixture of $50 \% \mathrm{O}_{2}$ and $50 \% \quad \mathrm{~N}_{2} \mathrm{O}$ under spontaneous breathing, and sevoflurane added step wisely up to $5 \%$ until unconsciousness was gained. A $25 \mathrm{G}$ intravenous line was inserted to a superficial vein in the forearm, and after administration of atropine $0.1 \mathrm{mg}(\approx 6 \mathrm{mg} / \mathrm{kg})$, infusion of remifentanil was started at a rate of $0.5 \mathrm{mcg} / \mathrm{kg} / \mathrm{min}$. A bolus dose of atracurium $10 \mathrm{mg}(\approx 6 \mathrm{mg} / \mathrm{kg})$ was given and the boy was ventilated with a facemask for three minutes with $100 \% \mathrm{O}_{2}$. Direct laryngoscopy with \#1 Macintosh blade enabled good view of the vocal cords (Cormack score 1) and endotracheal intubation with an uncuffed $5 \mathrm{~mm}$ internal diameter endotracheal tube was facilitated.

After fixation of the endotracheal tube the eyes were taped, a wire thermometer was inserted in the rectum and the boy was wrapped with an air heated blanket connected to a forced air warmer set at $38^{\circ} \mathrm{C}$. A bolus dose of $1 \mathrm{mg}$ morphine $(\approx 60 \mathrm{mcg} / \mathrm{kg}$ ) was injected and the sevoflurane concentration was lowered to 0.3 in the expired air.

The operation lasted 50 minutes, during which there was no significant bleeding; the heart rate was stable at $80 \mathrm{beat} / \mathrm{min}$ and blood pressure at $90 / 60 \mathrm{mmHg}$.

At the end of the procedure the remifentanil infusion was stopped, sevoflurane withhold and the boy was ventilated with $100 \% \mathrm{O}_{2}$ for $5 \mathrm{~min}$. Atropine $0.2 \mathrm{mg}$ and neostygmine $0.5 \mathrm{mg}$ were given to prevent residual neuromuscular blockade. Four min later the boy gained consciousness and began to breathe spontaneously. The trachea was extubated and the boy was taken to the post anesthesia care unit (PACU).

During 70 minutes of PACU stay the boy was stable, did not experience events of desaturation, hypotension, nausea or vomiting. He was given two more doses of 1 mg morphine for analgesia, and discharged to the pediatric surgery department with Aldrete score 4.

During the next day in the pediatric surgery department he gradually started ingesting soft food, and was sent home 24 hours later with no signs of bleeding.

\section{Discussion}

As for now, the literature lacks reports of anesthetic management of children with CDG I-x, and this report may provide clinicians an option for safe anesthetic management. The use of propofol based anesthesia, as well as the use of longer acting muscle relaxants and opioides may result in prolonged eduction and undesirable adverse reactions.

CDGs, formerly known as carbohydrate-deficient glycolprotein syndromes, consist of at least 18 genetic defects in the glycosylation of proteins. The CDGs comprise two groups. The first group, CDG I, includes defects in the N-glycan assembly localized in the cytosol and the endoplasmic reticulum (ER) including type I-a to I-l; CDG II comprises disorders in the processing of glycoproteins in the ER and Golgi apparatus accounting for types II-a to II-f [1]. Untyped cases are labeled CDG-X until the gene and its protein will be identified [2]. IEF of serum transferrin remains the most powerful screening test for CDG, although not all types can be detected by this assay. A type I IEF pattern (observed in CDG I) is characterized by a decrease of tetrasialotranferrin and an increase of di- and asialotransferrin bands, whereas a type 2 pattern (observed in CDG II) shows in addition and increase of tri- and monosialotransferrin [3].

The reports of various types have emphasized marked phenotypical variability of this multisystemic group of disorders [4]. The clinical presentation of CDG may include growth retardation, short limbs, delayed puberty and hypogonadism; hypertrophic or dilated cardiomyopathy and pericarditis [5] ataxia, psychomotor retardation, epilepsy, hypotonia, cerebral and cerebellar atrophy; bleeding tendency due to a decrease in Factors VII levels, and/or thromobosis due to decreased clotting factor $\mathrm{XI}$, protein $\mathrm{S}$ and protein $\mathrm{C}$; liver fibrosis and cirrhosis, chronic diarrhea, vomiting and protein-losing enteropathy; nephrotic syndrome; ichthyosis, abnormal fat pads and inverted nipples; and strabismus, abnormal eye movements, optic nerve atrophy, coloboma and retinitis pigmentosa.

IEF of serum transferring remains the most powerful screening test for $\mathrm{CDG}$, although not all types can be detected by this assay. A type I IEF pattern (observed in CDG I) is characterized by a decrease of tetrasialotranferrin and an increase of di- and asialotransferrin bands, whereas a type II pattern (observed in CDG II) shows in addition and increase of tri- and monosialotransferrin.

This case report presents a possible anesthesia regimen to be used in patients with CDG Ix scheduled for surgery. Previous report [6] regarding anesthetic management of CDG I patient described a sevoflurane-propofol-sufentanyl based regimen. Since propofol tends to lower systolic blood pressure and cardiac output, it posses a potentially negative effect in hemodynamiclly unstable patients and in patients with severe structural 
heart disease or congestive heart failure. Cardiac involvement contributes significantly to morbidity and mortality and probably to sudden death in CDG patients, thus propofol may better be circumvented in these patients.

The use of remifentanil is proven to reduce postoperative nausea and vomiting [7] and to provide faster recovery and shorter time to tracheal extubation compared with fentanyl or sufentanyl in tonsillectomy and adenoidectomy in the pediatric population [8]. Since remifentanil is metabolized by non specific esterases [9], its metabolism could be less affected by hepatic dysfunction resulting from CDG. We believe the use of a nitrous oxide-remifentanil based anesthesia regimen may be preferred for CDG I-x patients requiring general anesthesia.

\section{REFERENCES}

[1] A. E. Collins and D. M. Ferriero, "The Expanding Spectrum of Congenital Disorders of Glycosylation," The Journal of Pediatrics, Vol. 147, No. 6, 2005, pp 728-730. doi:10.1016/i.jpeds.2005.08.064

[2] J. Leonard, S. Grunewald and P. Clayton, "Diversity of Congenital Disorders of Glycosylation," Lancet, Vol. 357, No. 9266, 2001, pp. 1382-1383. doi:10.1016/S0140-6736(00)04618-3

[3] H. Engelhardt, M. Staudt, A. Hassler, et al., "Carbohy-
drate-Deficient Glycoprotein Syndrome Type 2," Journal of Inherited Metabolic Disease, Vol. 22, No. 2, 1999, pp. 192-193. doi:10.1023/A:1005422610091

[4] T. Marquardt and J. Denecke, "Congenital Disorders of Glycosylation: Review of Their Molecular Bases, Clinical Presentations and Specific Therapies," European Journal of Pediatrics, Vol. 162, No. 6, 2003, pp. 359-379.

[5] J. Kusa, A. Pyrkosz, A. Skiba and M. Szkutnik, "Cardiac Manifestations of Carbohydrate-Deficient Glycoprotein Syndrome," Pediatric Cardiology, Vol. 24, No. 5, 2003, pp. 493-494. doi:10.1007/s00246-002-0399-6

[6] E. Meaudre, V. Meyrieux, I. Suprano, et al., "Anesthesia Considerations in Carbohydrate-Deficient Glycoprotein Syndrome Type I," Pediatric Anesthesia, Vol. 15, No. 10, 2005, pp. 905-906. doi:10.1111/j.1460-9592.2005.01671.x

[7] P. Rama-Maceiras, T. A. Ferreira, N. Molins, et al., "Less Postoperative Nausea and Vomiting after Propofol + Remifentanil versus Propofol + Fentanyl Anaesthesia during Plastic Surgery," Acta Anaesthesiology Scandinavica, Vol. 49, No. 3, 2005, pp. 305-311. doi:10.1111/j.1399-6576.2005.00650.x

[8] T. Loop and H. J. Priebe, "Recovery after Anesthesia with Remifentanil Combined with Propofol, Desflurane, or Sevoflurane for Otorhinolaryngeal Surgery," Anesthesia and Analgesia, Vol. 91, No. 1, 2000, pp. 123-129.

[9] J. P. Thompson and D. J. Rowbotham, "Remifentanil-An Opioid for the 21st Century," British Journal of Anaesthesia, Vol. 76, No. 3, 1996, pp. 341-343. 